\title{
In Situ Observation of Directed Nanoparticle Aggregation During the Synthesis of Ordered Nanoporous Metal in Soft Templates
}

\author{
Lucas R. Parent ${ }^{1}$, David B. Robinson ${ }^{2}$, Patrick J. Cappillino ${ }^{2}$, Patricia Abellan ${ }^{1}$, James E. Evans ${ }^{1}$, Nigel \\ D. Browning ${ }^{1}$, and Ilke Arslan ${ }^{1}$ \\ ${ }^{1}$ Pacific Northwest National Laboratory, P.O. Box 999, Richland, WA 99352, USA. \\ ${ }^{2}$ Sandia National Laboratories, 7011 East Ave. Livermore, CA 94550, USA.
}

Achieving controlled and reproducible material syntheses is a major goal in many fields of nanoscience, where material properties are strongly influenced by nanoscale dimensions and structure. Materials with tailored nanostructures and morphologies show enhanced properties in applications including catalysis, hydrogen storage, and electrochemical energy storage. For example, nanoporous palladium can absorb and release hydrogen much more rapidly than the bulk metal, and exhibits lower hysteresis [1,2]. Ordered nanoporous materials are usually grown from solution in the presence of an ordered soft template, such as a concentrated surfactant or polymer solution that forms a close-packed micelle array or bicontinuous structure. However, most such materials are invented through painstaking iterations of separate synthesis and ex situ characterization steps, searching for conditions where the product reacts enough to obtain good connectivity around the micelles, and form large, sturdy structures, but does not overgrow the template, or otherwise disrupt it. This methodology is fundamentally limited in that the growth stages and mechanisms that occur during synthesis can only be potentially inferred from the final synthetic structure using ex situ characterization, and might be missed all together. By using in situ liquid (scanning) transmission electron microscopy ((S)TEM) to directly observe nanomaterial growth in real-time, we are able to gain unprecedented insight into the relationship between synthesis conditions and product evolution, facilitating a mechanistic approach to tailored nanomaterial development.

In the work reported here, we use a commercially available in situ liquid stage and STEM to observe the electron beam induced growth of nanoporous palladium structures in a solvated block copolymer (BCP) template under various synthesis conditions, and ultimately determine a refined synthesis procedure that yields extended structures with ordered pores. We find that after sufficient drying time of the casting solvent (tetrahydrofuran, THF), the BCP assembles into a rigid, cylindrical micelle array with a high degree of short-range order, but poor long-range order (figure 1, 15-minute ambient air drying). Upon slowing the THF evaporation rate using a solvent-vapor anneal (SVA) step, the long-range order is greatly improved (figure 2, 60-minute SVA). The electron beam induces nucleation of small particles in the aqueous phase around the micelles. The small particles then flocculate and grow into denser structures that surround, but do not overgrow, the micelles, forming an ordered nanoporous structure. The microscope observations reveal that disordered pores result from micelle disorder prior to metal reduction, which we could mitigate through a solvent-vapor annealing step prior to reduction. More broadly, this work demonstrates a new approach to rapid material development in which synthesis and characterization are simultaneous, and growth conditions can be modified with real-time feedback.

[1] D. B. Robinson et al., International Journal of Hydrogen Energy, 34 (2009) 5585-5591.

[2] P. J. Cappillino et al., Journal of Materials Chemistry A, 1 (2013) 602-610. 
[3] This work was supported in part by the Presidential Early Career Award for Scientists and Engineers for IA. Sandia National Laboratories is a multi-program laboratory managed and operated by Sandia Corporation, a wholly owned subsidiary of Lockheed Martin Corporation, for the U.S. Department of Energy's National Nuclear Security Administration under contract DE-AC04-94AL85000. The Pacific Northwest National Laboratory is operated by Battelle for the U.S. Department of Energy under contract DE-AC05-76RL01830.
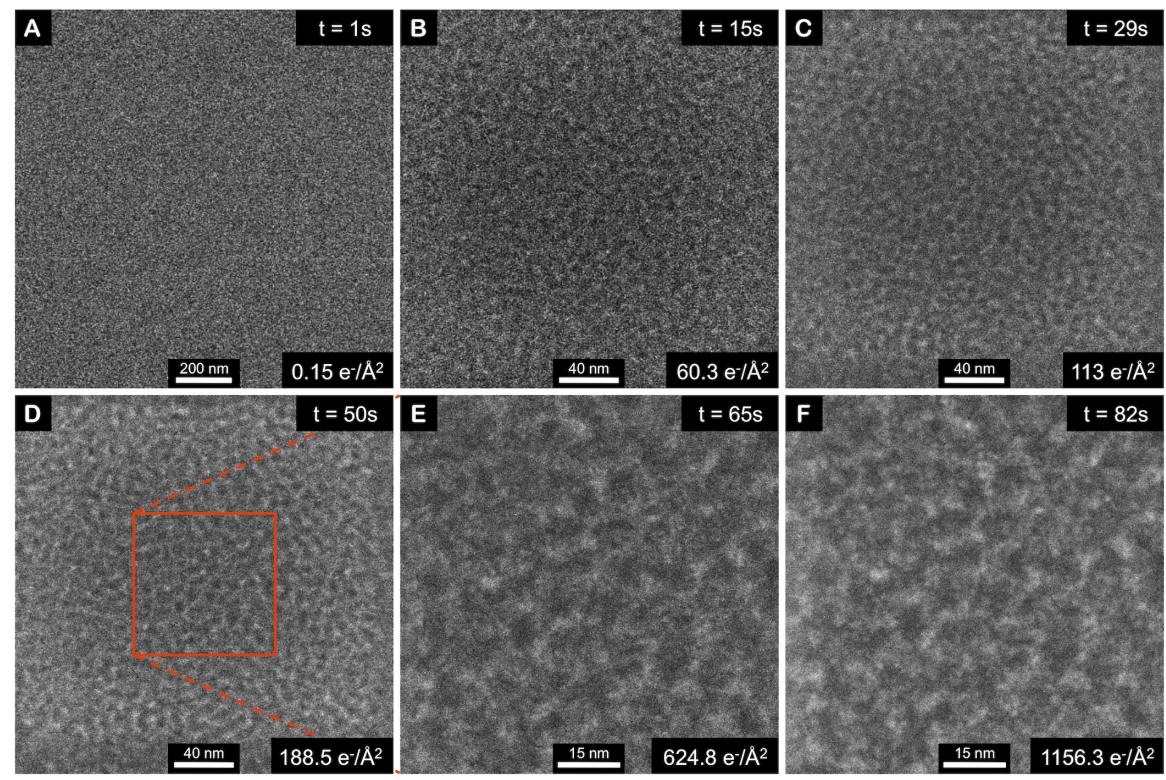

Figure 1: DF STEM images of growth of Pd nanostructure by e-beam induced reduction of Pd-salts within the BCP template after 15-minute ambient air exposure. Reprinted with permission from Chem. Mater. 2014, 26,1426-1433, DOI: 10.1021/cm4035209. Copyright 2014 American Chemical Society.
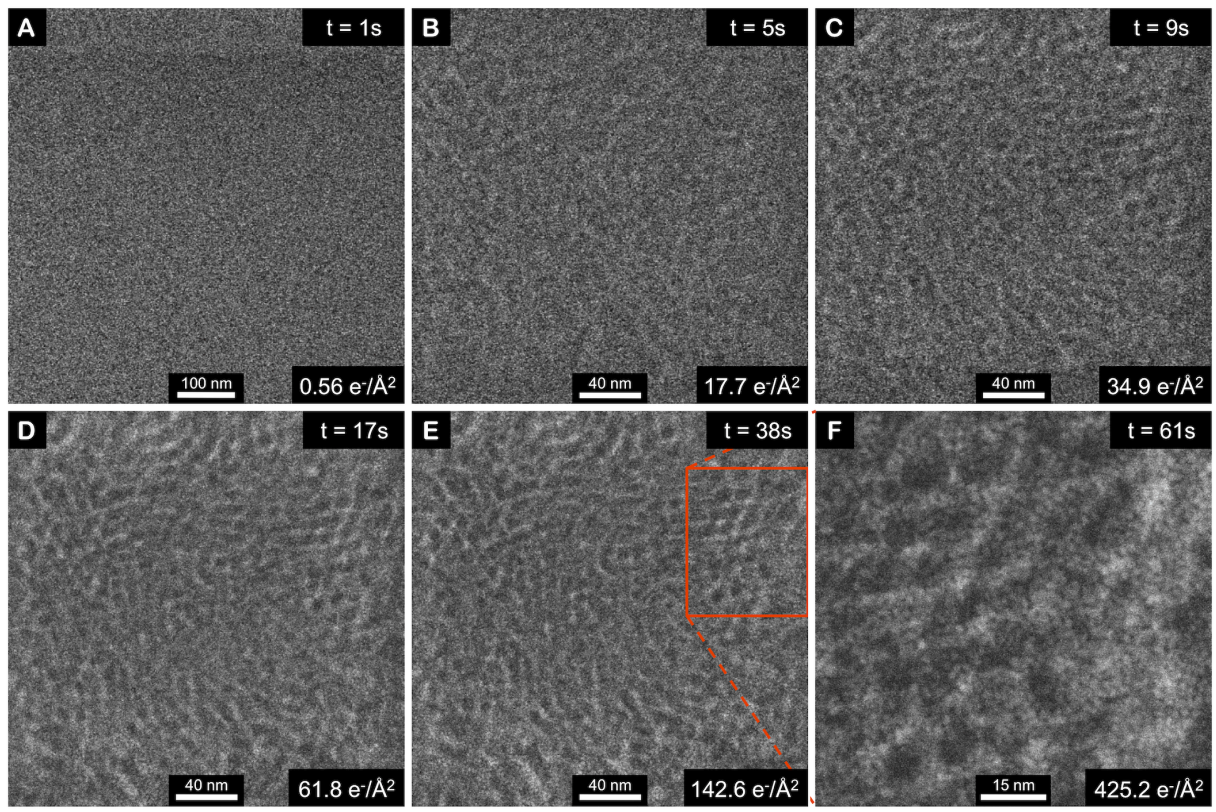

Figure 2: DF STEM images of growth of Pd nanostructure by e-beam induced reduction of Pd-salts within the BCP template after 60-minute solvent-vapor anneal. Reprinted with permission from Chem. Mater. 2014, 26, 1426-1433, DOI: 10.1021/cm4035209. Copyright 2014 American Chemical Society. 